\title{
Multiscale Poly-( $\epsilon$-caprolactone) Scaffold Mimicking Non-linearity in Tendon Tissue Mechanics
}

\author{
Brittany L. Banik ${ }^{1}$ - Gregory S. Lewis ${ }^{2}$. Justin L. Brown ${ }^{1}$
}

Received: 8 October 2015 / Accepted: 6 January 2016/Published online: 25 January 2016

(C) The Regenerative Engineering Society 2016

\begin{abstract}
Regenerative medicine plays a critical role in the future of medicine. However, challenges remain to balance stem cells, biomaterial scaffolds, and biochemical factors to create successful and effective scaffold designs. This project analyzes scaffold architecture with respect to mechanical capability and preliminary mesenchymal stem cell response for tendon regeneration. An electrospun fiber scaffold with tailorable properties based on a "Chinese-fingertrap" design is presented. The unique criss-crossed fiber structures demonstrate nonlinear mechanical response similar to that observed in native tendon. Mechanical testing revealed that optimizing the fiber orientation resulted in the characteristic "S"-shaped curve, demonstrating a toe region and linear elastic region. This project has promising research potential across various disciplines: vascular engineering, nerve regeneration, and ligament and tendon tissue engineering.
\end{abstract}

\section{Lay Summary}

A novel scaffold created from biodegradable fibers and incorporating unique criss-cross fiber geometry was synthesized. The scaffold recapitulated the complex non-linearity in mechanics of tendon and ligament tissues. Furthermore, the scaffold supported the growth of mesenchymal stem cells, and preliminary data

Justin L. Brown

jlbbio@engr.psu.edu

1 Department of Biomedical Engineering, The Pennsylvania State University, 205 Hallowell Building, University Park, PA 16802, USA

2 Department of Orthopaedics and Rehabilitation, Penn State College of Medicine, Hershey Medical Center, 500 University Dr, Hershey, PA 17033, USA suggests that the scaffold geometry encourages the differentiation of mesenchymal stem cells towards tendon.

Keywords Tendon $\cdot$ Regenerative medicine $\cdot$ Bioinstructive scaffold $\cdot$ Electrospinning $\cdot$ Poly- $(\epsilon$-caprolactone $)$

\section{Introduction}

Tendon injuries are often debilitating and painful and can significantly decrease the quality of life for patients that have received an unsuccessful treatment or incurred incomplete healing. With $30-50 \%$ of sports-related injuries in the USA being related to acute tendon injuries [1], it is not surprising that total tendon and ligament injury costs were estimated as high as $\$ 30$ billion annually in the USA in 2000 [2]. Tendons are integral in day-to-day activities and function as the fibrous connective tissue that joins muscles to bones ultimately aiding in joint stability, promoting motion, transmitting forces, and storing and releasing energy [3]. Tendon injuries have relatively poor healing capabilities due to the limited cell number within the tissue and poor vascularization [4]. Without adequate treatment, unorganized collagen and scarring form during healing, which compromise the overall critical tissue mechanics [5]. There is an inherent need for improved tendon and ligament scaffolds with rising economic burdens and healthcare costs and numerous populations affected, including the aging population, military, space, recreational and elite athletes, and areas of trauma, congenital defects, and tumors.

Current tendon reconstruction methods continue to fail to provide full joint mobility and often result in mechanical failure, wear, or infection [3]. The hierarchical crimped and aligned architecture of collagen fibers in tendons translate to high tensile strength tissues that can withstand continuous force. Autografts provide benefits of initial mechanical 
stability, highest stiffness in tendon biomechanical constructs available, and reduction in the chance of late rupture; however, issues include surgical and donor site morbidity, finite availability, and inadequate revascularization [3, 6]. Allografts, which demonstrate an advantage of no donor site morbidity and similar mechanical strength compared with autografts, have associated challenges including potential for disease transmission and bacterial infections, a limited donor pool, and an increased failure rate (up to four times higher) for young, active patients [7]. Thus, the market demand promotes synthetic scaffolds as an ideal solution within the regenerative engineering field [6]. Regenerative engineering is the convergence of advanced biomaterials science with stem cell and developmental biology to repair, replace, or regenerate tissues to restore structure and function [8-10]. The area of regenerative engineering has the potential to be transformative in translational and clinical applications [10] because it converges multiple systems critical to effective cell-based therapies and tissue-related replacements. The natural, dynamic, cell microenvironment defines a complex cell niche with the challenge to balance cellular cues within the scaffold matrix with cellular response and function. Understanding cellmatrix interactions and how they apply to the various tissues will be key. Regenerative engineering has shown promising results in areas of commercial skin products, sports injury cartilage damage, and bladder dysfunction treatments [8]. The presented research falls into the pioneering regenerative engineering field in the area of tendon tissue engineering.

Synthetic scaffolds allow for tailorability, reproducibility, and low immunogenicity risks [6]. However, synthetic regenerative medicine-based treatment options for tendon injuries still fall short in areas of host tissue integration, mechanical stability, long-term performance, physiological mechanical response of natural tendon, and balancing tissue remodeling and biodegradation of the material [2]. New therapeutic solutions must provide sufficient early biomechanical strength to withstand weight-bearing motions during rehabilitation and introduction to patient day-to-day activities, serve as a scaffold to facilitate cell adhesion, differentiation, and development, remain biocompatible to the host, and be integrative to the graft connection points.

Regenerative medicine is structured around three main components: stem cells, biochemical factors such as growth factors or cytokines, and biomaterial scaffolds to repair complex tissues. The scaffold architecture presented to cells has been demonstrated to play a critical role in cellular responses, such as proliferation and differentiation, ultimately establishing the "cell niche" and affecting tissue regeneration, reparation, or replacement. This project presents a prime achievement within the soft regenerative medicine field: a novel fiber scaffold structure with tailorable mechanical properties based on a "Chinese-fingertrap" design. The scaffolds are fabricated from an innovative electrospinning technique with a hierarchical perspective: cell instructive nano-structure, crisscrossed micro sub-structure, and macro bulk shape and mechanical response. Electrospinning has become a popular fabrication technique for tendon scaffold development based on resulting similarities to the extracellular matrix (ECM) and the ability to modify the electrospun output based on material choice (e.g., synthetic or natural), spinning and processing conditions, and scaffold requirements (e.g., mechanical anisotropy, fiber orientation, porosity, cellular response, etc.). The ECM-like fibers produced by electrospinning provide a microenvironment for cells with a high aspect ratio, surface area, permeability, and porosity [11]. A number of researchers have utilized electrospinning to attempt to exploit the electrospun fiber benefits for regenerative medicine applications [12-15].

The proposed design purpose includes developing the scaffold architecture to mimic the non-linear stress-strain curve of natural tendon and utilizing the nanofiber geometry to instruct cells [16-18] towards tenogenesis. The results of this project have a broad impact and promising project potential with the opportunity to span across various regenerative medicine applications, including vascular engineering, nerve regeneration, and ligament and tendon tissue engineering, to improve the clinical outcome and patient quality of life.

\section{Materials and Methods}

\section{Fabrication of Electrospun Scaffolds}

Poly-( $\varepsilon$-caprolactone) (PCL; MW 80,000; Sigma-Aldrich), acetone (BDH Chemicals), and glacial acetic acid (EMD Millipore) were used as received. A total polymer solution of $20(w / v)$ PCL in acetone and glacial acetic acid (V1/V2, $80 / 20$ ) was prepared by mixing with heat and stirring for $\sim 3 \mathrm{~h}$ on a hot plate to ensure complete dissolution. The polymer solution was injected through a single nozzle spinneret (18-gauge needle) at a constant flow rate of $1.5 \mathrm{~mL} / \mathrm{h}$ with an accelerating voltage of $15 \mathrm{kV}$ applied. The target distance was $26 \mathrm{~cm}$ from the needle tip to the rotating rod target setup. Scaffold synthesis was modified from a basic electrospinning method by using a rotating rod target and magnets. Solution components and electrospinning conditions are organized in Table 1 with the design setup shown in Fig. 1.

To create the tube-shaped scaffolds with bi-axially aligned fibers, the $20(w / v)$ PCL polymer solution was spun with an innovative electrospinning setup: Fibers were targeted to a dual directional oscillating rod. The fiber alignment was controlled with magnets flanking the target area, which pulled fibers to be aligned parallel to the rod, while the rotational speed was changed to control fiber alignment and ultimately to regulate the 
Table 1 Electrospinning conditions for scaffold synthesis

\begin{tabular}{ll}
\hline Polymer solution & $20 \% \mathrm{PCL}, 80: 20$ glacial acetic acid:acetone \\
Voltage, flow rate & $15 \mathrm{kV}, 1.5 \mathrm{~mL} / \mathrm{h}$ \\
Target distance & $26 \mathrm{~cm}$ \\
Humidity & $50-60 \%$ \\
Temperature & $21-24^{\circ} \mathrm{C}$ \\
Speed of rotation & $184-328 \mathrm{rpm}$ \\
\hline
\end{tabular}

mechanical properties of the scaffold. Samples were evaluated with environmental scanning electron microscopy (ESEM) to confirm fiber alignment.

\section{Scanning Electron Microscopy}

The Quorom EMS 150 Sputter Coater was used to deposit a conductive coating of 50 angstroms of gold for $30 \mathrm{~s}$ on the polymer fiber scaffolds to reduce charging. An environmental scanning electron microscope (ESEM FEI, Quanta 200) was used in low vacuum mode to acquire images for fiber alignment. ImageJ was utilized to determine the angles of orientation for individual fibers by manually drawing lines on the scaffold SEM images. Lines were drawn until the fibers presented on the image were exhausted. Histograms were plotted to represent fiber alignment for each speed with $n=100$ for $184 \mathrm{rpm}$, $n=100$ for $216 \mathrm{rpm}, n=100$ for $264 \mathrm{rpm}$, and $n=75$ for $328 \mathrm{rpm}$.

\section{Mechanical Properties of Electrospun Scaffolds}

For tensile testing, electrospun scaffolds were mounted onto 3D-printed t-glase stubs by securing both ends of the scaffold with tightly wound parafilm and a zip-tie. It was determined that a notched stub design provided the resistance necessary to prevent the zip-tie from sliding during mechanical testing experiments; the parafilm acted as a barrier between the ziptie grip pattern and the PCL scaffold so that the zip-tie did not puncture or tear the scaffold when cinched. The parafilm, ziptie, and notched stub combination prevented slipping of the scaffold and zip-tie during testing and also allowed the scaffold to break more realistically in the middle of the scaffold rather than on the connecting ends near the stub and scaffold providing a better indication and readout of the scaffold failure in tension.

The stub design was created in Solidworks, processed in Repetier (open-access 3D-printing software), and printed with a SeeMeCNC Rostock MAX v2 Desktop 3D-printer. The stubs on either end of the scaffold were inserted into the pressurized grips on an Instron 5960 Dual Column Tabletop tensile machine. A 1-kN load cell was used with a crosshead speed of $10 \mathrm{~mm} / \mathrm{min}$. Prior to tensile tests, the following scaffold dimensions were recorded in order to calculate the scaffold thickness: mass (Sartorius CP 124S Balance), length (Brown \& Sharpe calipers), and diameter (Brown \& Sharpe calipers). Scaffold thickness was determined by using the measured scaffold dimensions in annulus area calculations:

\section{Scaffold annulus area}

$$
=\text { mass } /\left(\text { length }{ }^{*} \text { density }\right), \text { where } \rho_{P C L}=1.135 \mathrm{~g} / \mathrm{cm}^{3}
$$

Scaffold annulus area $=A-A_{r}$, where $A=\pi\left(\frac{d_{\text {outer }}}{2}\right)^{2}$ and $A_{r}=$ $\pi\left(\frac{d_{\text {imer }}}{2}\right)^{2}$ with $d_{\text {inner }}=d_{\text {outer }}-2 t$ and $t=$ thickness.

Gauge length (Brown \& Sharpe calipers) was measured as an input value for the tensile testing software. The load was zeroed to remove fixturing loads and an internal system signal calibration was performed before each test to balance the load. Output values were load, time, stress, and strain. Stress was calculated by load/annulus area.
Fig. 1 Electrospinning setup for tendon scaffold synthesis. a The collecting target is a rod rotating twice counterclockwise and then switching directions and rotating twice clockwise. b Device mechanism used to rotate the collecting rod target a)
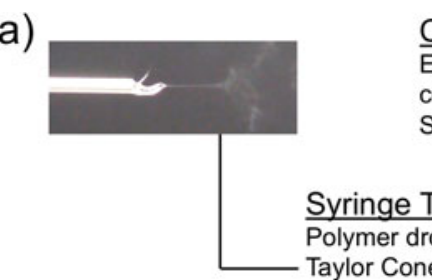

Charged Ejected Fiber

Electrostatic repulsion counteracts surface tension Solvent evaporates

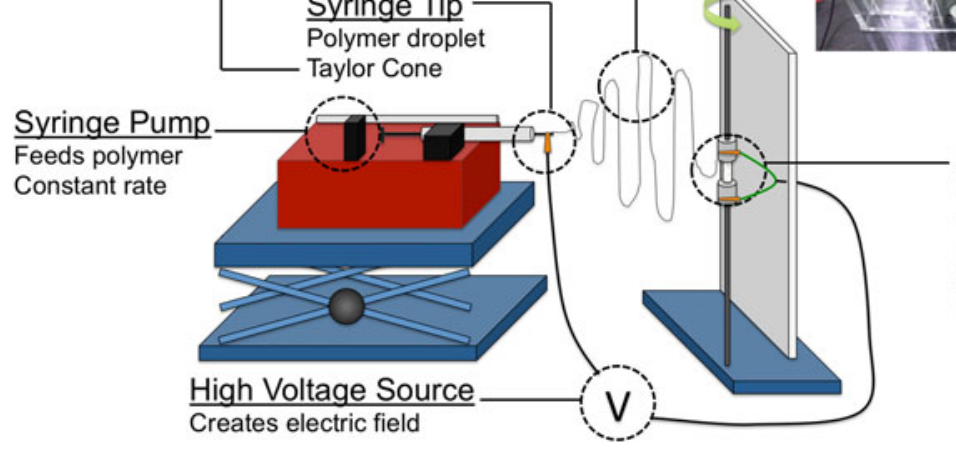

Collecting Target

Conductive

Grounded source

Magnets orient fibers

Spinning creates tube

Fibers align to rod axis 


\section{Distinction of Elastic Moduli and Toe Regions}

The elastic moduli were determined based on the data output from the stress-strain curves by taking the slope of the line through ten consecutive points throughout the stress-strain values. The maximum value was considered the elastic modulus found in the linear region of the stress-strain curve for each scaffold. Each calculation was reviewed to ensure the elastic modulus was not determined from an obscure portion of the curve.

To define the maximum boundary of the toe region quantitatively, a line was generated with the slope corresponding to the elastic modulus and intersecting the inflection point of the linear elastic region on the stress-strain curve. The inflection point was based on the maximum of the first derivative. The $\mathrm{x}-$ intercept of this line was used to define the maximum of the toe region for the curve. This value was considered the upper boundary of the toe region with the lower value assumed as $0 \%$. All calculated values were validated with visual inspection of the stress-strain graphs. For elastic moduli and toe region samples, $n=5$.

\section{Cell Culture and Fluorescence Microscopy}

Human mesenchymal stem cells (hMSCs; Lonza, Biosciences, passage 7) were maintained in alpha-modified minimum essential medium ( $\alpha$-MEM), $10 \%$ fetal bovine serum (FBS), and $1 \%$ penicillin streptomycin (Pen-Strep). The hMSCs were cultured at $37^{\circ} \mathrm{C}$ with $5 \% \mathrm{CO}_{2}$ in a humidified incubator. The scaffolds were sterilized with UV light for 5 min. hMSCs were micromass seeded on the scaffolds by pelleting the cells, resuspending the cells in $100 \mu \mathrm{l}$ of media, and placing a $100-\mu l$ droplet of cells on the scaffolds for $30 \mathrm{~min}$ before filling the well with the appropriate media amount. The cells were grown for 7 days.

Immunostaining was performed to stain F-actin and double-stranded DNA in cell nuclei. In brief, cells were fixed in $3.7 \%$ paraformaldehyde for $15 \mathrm{~min}$ and incubated with a permeabilization buffer ( $3 \%$ bovine serum albumin and $0.1 \%$ Triton X-100 in 1X phosphate-buffered saline (PBS)) for $45 \mathrm{~min}$ at room temperature. Cells were incubated with permeabilization buffer containing a 1:1000 dilution of Phalloidin 565 to fluorescently label actin and a 1:5000 dilution of DAPI to stain the nuclei for $30 \mathrm{~min}$. Immunostained samples were imaged directly with a $20 x$ objective on a Leica DM5500 upright microscope (Leica Microsystems, Buffalo Groove, IL).

\section{RNA Extraction and RT-PCR Quantification}

Human mesenchymal stem cells (Lonza, Biosciences, passage 8) were seeded on a glass coverslip (control) and proposed scaffold design produced at $264 \mathrm{rpm}$ (fiber

size $=271 \mathrm{~nm}$ ) and cultured for 16 days. RNA extraction and real-time polymerase chain reaction (RT-PCR) were performed with the Qiagen $\mathrm{RT}^{2}$ ProfilerTM PCR Array Human Mesenchymal Stem Cells kit (cat. no. 330231 PAHS-082ZA) $(n=1)$. The RNA extracts were purified with the Qiagen RNeasy ${ }^{\circledR}$ Plus Mini Kit (cat. no. 74134) before running RT-PCR. cDNA was used as a template for real-time PCR with SYBR Green (Qiagen RT [2] SYBR Green ROX qPCR Mastermix, cat. no. 330520) and gene specific primers identified in the kit in an Applied Biosystems ${ }^{\circledR} 7300$ thermocycler (Huck Institutes of the Life Sciences, The Pennsylvania State University). Gene expression levels were standardized to the internal system controls and normalized to expression in control glass coverslips. The housekeeping genes used as controls on the PCR plate were: ACTB, B2M, HPRT1, and RPLP0. To be considered relevant, changes had to be measured at a level below $35 \mathrm{Ct}$.

\section{Statistical Analysis}

Outliers were removed based on the 1.5 interquartile range method. Statistical significance was determined by a oneway ANOVA and a subsequent Tukey's post hoc test with a critical value of $p<0.05$.

\section{Results}

\section{Electrospun Scaffold Design}

The proposed scaffold design is shown in Fig. 2a. While the structure created in the proposed electrospinning process is not woven in architecture like the Chinese-fingertrap puzzle,

a)

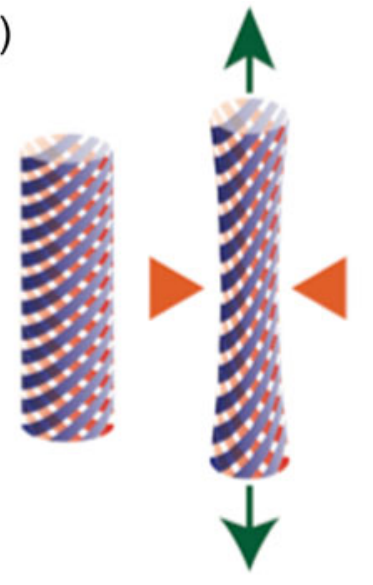

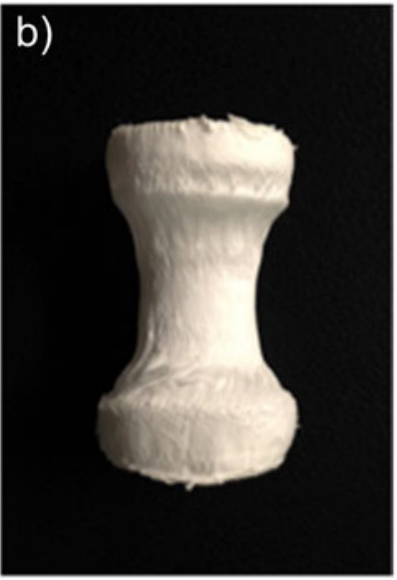

Fig. 2 Proposed tendon scaffold. a Cartoon of the tendon scaffold illustrating the Chinese-fingertrap design — when the scaffold is pulled on the ends, a change in length is associated with a change in radius. b Image of final electrospun PCL tendon scaffold 
it is similar with overlapping layers of bi-axially aligned fibers. The final scaffold is shown in Fig. 2b, with magnets placed $2.8 \mathrm{~cm}$ apart during the electrospinning process (Fig. 1). Scaffolds were detached as complete 3D tube structures by removing the magnets and sliding the scaffold off the rod in one piece. Expected size dimension for scaffolds spun on a rod with a diameter of $6.35 \mathrm{~mm}$ were length $=2.8 \mathrm{~cm}$, thickness of tube wall $=0.0691 \mathrm{~mm} \pm 0.0158(n=20)$.

\section{Fiber Alignment}

Electrospun scaffolds were produced via the novel rotating rod setup described previously in Fig. 1 at various speeds184 rotations per minute $(\mathrm{rpm} ; 0.061 \mathrm{~m} / \mathrm{s}), 216 \mathrm{rpm}(0.071 \mathrm{~m} /$ s), $264 \mathrm{rpm}(0.087 \mathrm{~m} / \mathrm{s})$, and $328 \mathrm{rpm}(0.108 \mathrm{~m} / \mathrm{s})$-which were validated for each scaffold. Electrospinning conditions were kept constant with humidity and temperature varying slightly between tests. Fiber alignment and physical characteristics were determined through scanning electron microscopy. Fibers were smooth and continuous. To assess fiber alignment based on the speed of rotation, lines were drawn on 75-100 fibers to determine the angle of alignment. Figure 3 illustrates representative histograms for fiber alignment at each speed. For the 184- and 216-rpm scaffolds, a single peak is observed for fiber alignment, suggesting that overall fiber alignment is parallel to the rod's vertical axis $-57 \%$ of fibers fell between $60^{\circ}$ and $90^{\circ}$ for $184 \mathrm{rpm}$ and $54 \%$ of fibers fell between $80^{\circ}$ and $100^{\circ}$ for $216 \mathrm{rpm}$. For the 264- and 328-rpm speeds, the fiber alignment peaks begin to broaden, with two distinct peaks beginning to develop in the $328 \mathrm{rpm}$ scaffolds. Fibers span $20-100^{\circ}$ for alignment in the $264 \mathrm{rpm}$ samples with $73 \%$ of the fibers falling in this range. With the 328-rpm scaffolds, fibers begin to fall into two peaks: $56 \%$ of fibers with fiber alignment between $50^{\circ}$ and $100^{\circ}$ and $24 \%$ between $110^{\circ}$ and $140^{\circ}$. Figure 3 also demonstrates homogenous fiber diameters across the scaffolds of varying speeds without any
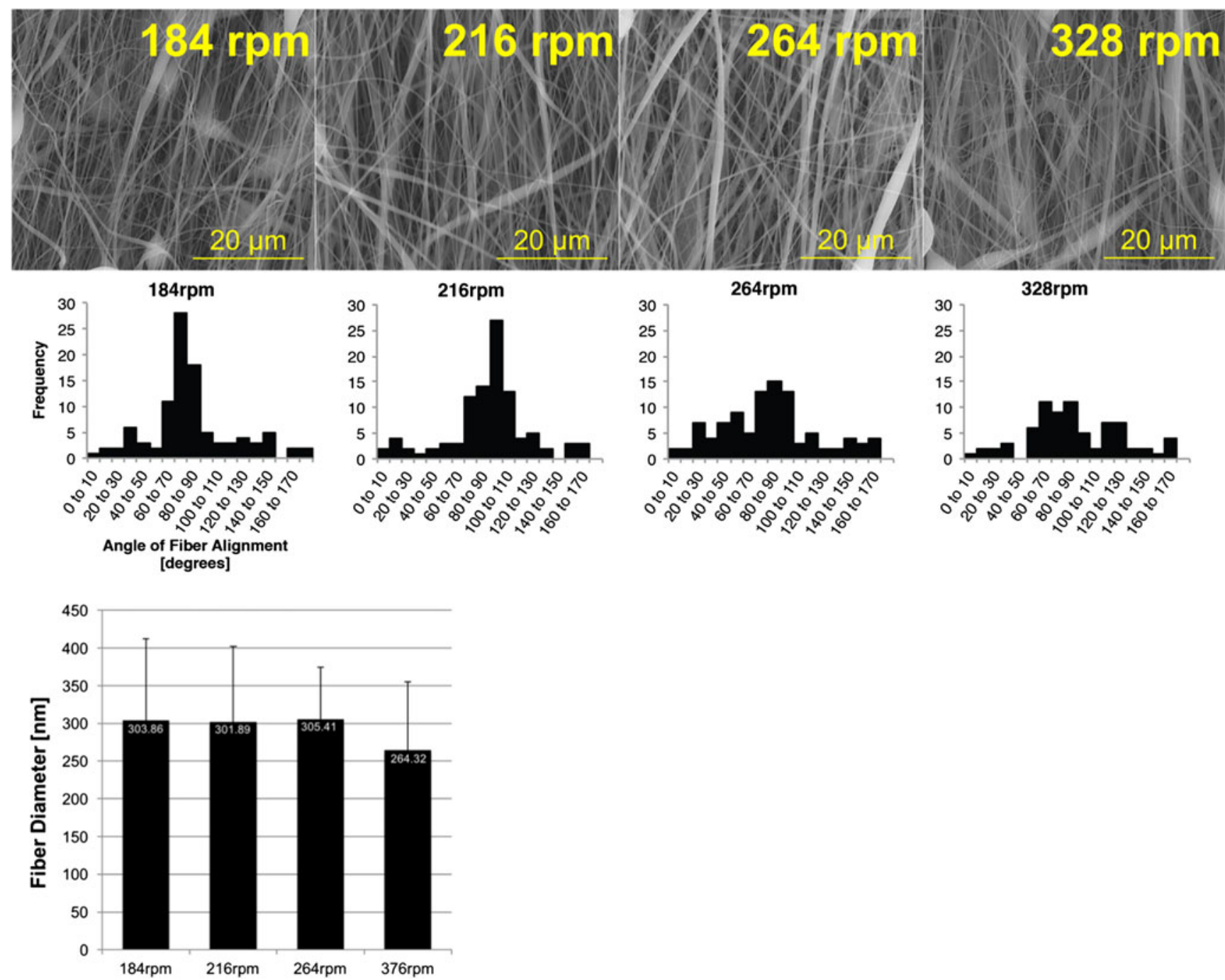

Fig. 3 SEM images and histograms representing fiber alignment on scaffolds produced at various speeds-184, 216, 264, and 328 rpm and corresponding fiber diameters 
significant differences amongst fiber groups. Factors that could play a role in scaffold variation include the need to clean the target during the electrospinning process and alignment of syringe-to-target during fabrication.

\section{Mechanical Response}

To analyze the scaffold mechanical response, the elastic moduli were calculated by determining the maximum slope from groups of 10 consecutive points within the linear portion of the stress-strain curves. The toe region maximum boundary value was the $\mathrm{x}$-intercept extrapolated from the inflection line with a slope defined by the elastic modulus. Figure $4 \mathrm{~b}$ illustrates a representative stress-strain graph for a 264 rpm scaffold. The toe region falls from 0 to $2.7 \%$, and the elastic modulus is $35.8 \mathrm{MPa}$.

\section{Elastic Moduli and Toe Region}

For the elastic moduli evaluations, shown in Fig. 5a, the various speeds demonstrated average values of $36.5 \pm 9.0 \mathrm{MPa}$ for $184 \mathrm{rpm}$ scaffolds, $24.4 \pm 6.6 \mathrm{MPa}$ for $216 \mathrm{rpm}$ scaffolds, $30.3 \pm 8.4 \mathrm{MPa}$ for $264 \mathrm{rpm}$ scaffolds, and $30.7 \pm 5.0 \mathrm{MPa}$ for $328 \mathrm{rpm}$ scaffolds. Natural tendon elastic moduli values can be as high as 1.2 GPa [19]. Significance was noted between 184 and $216 \mathrm{rpm}$ with a $p$ value of 0.028 for elastic moduli. For the toe region boundaries with the lower boundary starting at $0 \%$, the upper boundaries were as follows: for $184 \mathrm{rpm}, 5.3$ $\pm 2.8 \%$, for $216 \mathrm{rpm}, 6.7 \pm 3.0 \%$, for $264 \mathrm{rpm}, 3.4 \pm 1.1 \%$, and for $328 \mathrm{rpm}, 0.5 \pm 0.7 \%$ (Fig. 5b). The toe region for natural tendon typical falls in the range of $0-3 \%$. Significance was determined between 184 and $328 \mathrm{rpm}$ with a $p$ value of 0.012 and between 216 and $328 \mathrm{rpm}$ with a $p$ value of 0.001 for the toe region.

a)

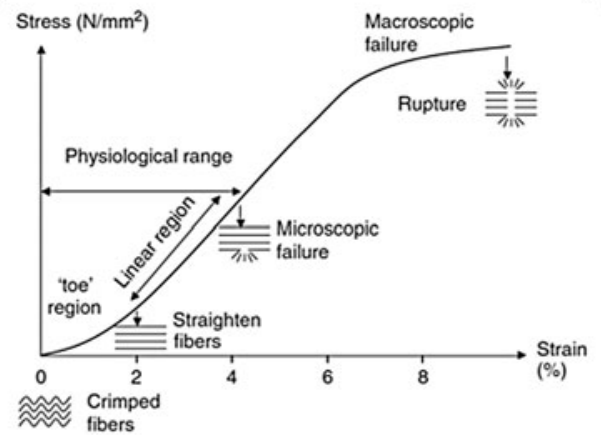

Fig. 4 Mechanical suitability of scaffolds. a Natural tendon stress-strain curve. The "toe region" from 0 to $3 \%$ is noteworthy. This represents the point at which the crimped collagen fibers are elongating and stretching until they are fully extended and reach the linear section of the curve [30] (Reprinted from Journal of Biomechanics 39(9), J. H.-C. Wang,

\section{Discussion}

Natural tendon tissue exhibits complexity in the hierarchical collagen structure, crimped architecture, and load-bearing mechanics. Scaffold requirements for synthetic options generally fall into categories of: physiological mechanical properties, structural support, degradation kinetics to balance rapid matrix remodeling and scaffold degradation, and bioactive and bioinstructive cues to improve healing response. Currently, no material can match the stiffness, ductility, non-linearity, and viscoelasticity [20] of natural tendon. Therefore, a replacement synthetic scaffold will need to harness the scaffold properties to direct the development and differentiation of cells to closely mimic natural tendon mechanical and biochemical properties to bring the graft closer to native physiological mechanical and cellular responses.

Our Chinese-fingertrap scaffold design (Fig. 2) was developed to improve the integration and mechanical properties of current synthetic tendon grafts by introducing a toe region in the tendon graft through the criss-crossed fibers. The alignment of the scaffold fibers during mechanical loading can be compared with the extinction of collagen fascicle crimping during loading of tendon [21]. The scaffold concept is also analogous to a Chinese-fingertrap puzzle in such that pulling on the bi-axially aligned fibers lengthens the trap and reduces the circumference of the ends to tighten the structure at the openings. The system lengthens as the diameter of the trap decreases. Literature suggests similarities between the Chinese-fingertrap design and characteristic connective tissue architecture by highlighting the fiber orientation and free gliding mobility as analogous to the fiber weave observed in the Chinese-fingertrap [22]. The fingertrap design also aids in mechanical means by providing stretch until a tension threshold is reached [23]. There is evidence in literature demonstrating the application of the Chinese-fingertrap design for sutures and fixation with benefits including allowance for vascularity,

b)

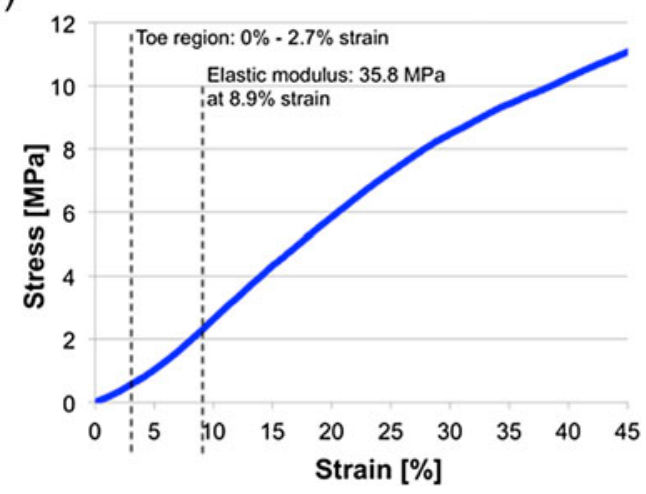

"Mechanobiology of Tendon," 1563-1582, 2006, with permission from Elsevier). b Representative stress-strain curve for scaffolds. The toe region falls from 0 to $2.7 \%$ with an elastic modulus of $35.8 \mathrm{MPa}$ for the represented scaffold, which is a scaffold spun at $264 \mathrm{rpm}$ 
Fig. 5 Tensile testing for scaffold mechanics. a Scaffold moduli based on rotational speed. $\mathbf{b}$ Scaffold toe region based on rotational speed. $\mathbf{c}$ Tendon scaffold mounted on 3D-printed stubs in tensile testing machine prior to test (left) and stretched scaffold during tensile testing experiment $($ right $)\left({ }^{*} p<0.05\right.$; $* * p<0.01 ; * * * p<0.001)$

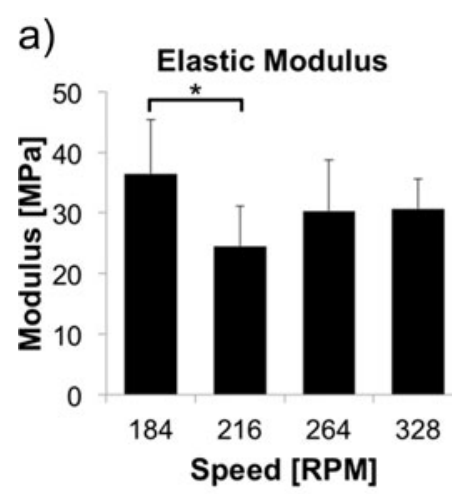

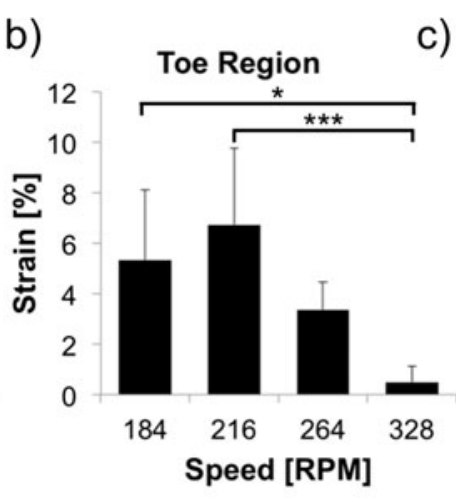

c)

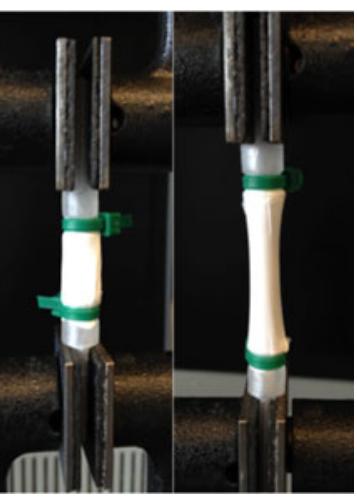

stable fixation, flexibility in dynamic suture tension response, and increased elongation before failure [24-26].

Figure 3 illustrates the ability to use the rotating rod mechanism and magnets to regulate fiber alignment as a function of rotation speed. Magnets were utilized to align the fibers parallel to the length of the rod while the rotations whip the fibers to angle along the rod during formation. The use of magnetic fields to align nanofibers during electrospinning has been demonstrated by Yang et al. [27] through generating aligned polymeric fibers by incorporating magnetic nanoparticles into the polymeric solution for the electrospinning process and spinning the nanofibers in the presence of a magnetic field; furthermore, Liu et al. [28] demonstrated the use of an external magnetic field near the collector target to achieve thick aligned fiber mats without the need for magnetic nanoparticles to be included in the polymer solution. In the current study, it is noted that fibers strongly aligned in the direction of the magnetic field for the slower rotational speeds and for the faster rotational speeds, 264 and $328 \mathrm{rpm}$, the histogram depicting the orientation of the nanofibers begins to broaden and show two distinct peaks. These characteristics suggest bi-axial fiber alignment. A Chi-square test was run to determine fiber randomness with the critical $\chi^{2}$ statistic value $<0.05$ indicating alignment. Based on the Chi-square test, all scaffolds exhibit alignment; the
184- and 216-rpm scaffolds display more aligned behavior than the 264- and 328-rpm scaffolds.

The complex mechanical physiological responses of natural tendon are difficult to replicate with synthetic scaffolds for various reasons, including: the significance of the toe region ( $0-3 \%$ strain under low stress), the high elastic modulus (upwards of 1.0 to $2.0 \mathrm{GPa}$ [29]), viscoelasticity, and the ability to withstand multiple dynamic cycles of use. Figures 4 and 5 illustrate the mechanical capability of the Chinese-fingertrap based scaffolds. Stress-strain data was obtained by normalizing measured load and displacement by scaffold geometry and is important because it describes the mechanical properties at the material level. The mechanical properties of the overall graft structure may be controlled by changing geometric parameters such as tube wall thickness. A typical stress-strain curve for native tendon is illustrated in Fig. 4a [30]. It is worthwhile to note the three main delineations of the curve - the low-strain curved toe region from 0 to $\sim 3 \%$ strain, linear elastic region extending to the yield point from $\sim 4$ to $7 \%$ strain, and macroscopic failure region beyond $\sim 8-10 \%$ with rupture occurring thereafter [3]. The proposed scaffold curve, shown in Fig. 4b, represents the important toe region target values and continues with the characteristic S-shaped curve of natural tendon. The toe region is critical, serving as the shock-absorbing feature of tendon mechanics to prevent tissue damage [31]. Within the toe region, the crimped
Fig. 6 Preliminary cell work a hMSCs exhibit early contact guidance phenomenon $\mathbf{b}$ hMSCs adhere and align along fibers forming a cell sheet in the direction of the bulk scaffold at a later timepoint (red, actin: Phalloidin; blue, nuclei: DAPI); arrows indicate direction of bulk scaffold
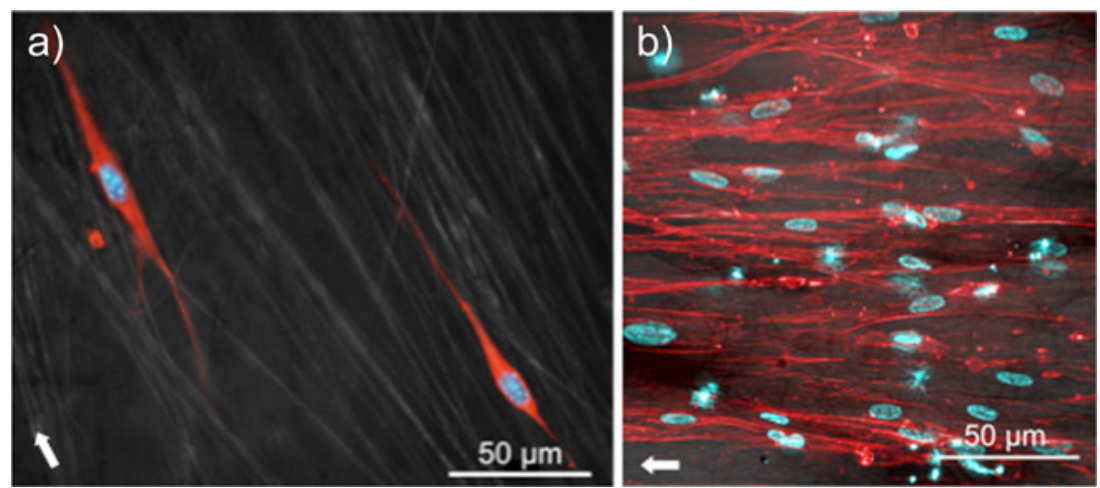
collagen fibers begin to elongate into the linear region. The linear region is an area where the collagen fibers exhibit resistance and align in the load direction. In the final region, the collagen fibrils begin to reveal structural damage as the collagen fibrils are stretched to extension. In Fig. 5, it is not surprising that the elastic moduli did not change significantly between scaffolds because the elastic moduli will only be as good as the material chosen if all the fibers are strained. A common limitation across synthetic scaffolds is reduced mechanical responses when compared with natural tissues. Many clinical rehabilitation protocols limit patient activity early after surgery, reducing the early functional requirements. Furthermore, in common rotator cuff repair surgery, patients could benefit from a scaffold that acts as a patch covering the repair site. This patch is not required to carry the full mechanical load of the rotator cuff and instead supplements the mechanical integrity and biologic healing.

Future work will include a long-term bioreactor culture period to improve mechanics and to evaluate the effects of cellular activity and ECM production on the elastic moduli. We hypothesize that an increase in elastic moduli will be exhibited due to the cells laying down ECM along the fibers presented via contact guidance cues. Future work will include characterization of additional scaffold properties such as ultimate strength. On the other hand, the toe region, a challenge to replicate in synthetic grafts, begins to more closely represent the native tendon mechanical response stress-strain curve based on the proposed design.

Cellular work demonstrates the ability for cells to adhere, grow, and proliferate on the presented scaffolds, which suggests limited to no effects of biomaterial cytotoxicity. Furthermore, PCL has been thoroughly vetted in previous literature studies to reveal no significant cytotoxicity $[32,33]$. Figure 6 illustrates the concept of contact guidance, in which cells align along the exposed topography. This will work in the favor of tendon regeneration because as the cells align along the fibers in a specific architecture, the scaffold is enhanced for biological integration and potentially for mechanical performance as the cells begin to lay down ECM. Additionally, further consideration was given to evaluate hMSC differentiation towards the tendon lineage on the $264 \mathrm{rpm}$ scaffold through RT-PCR, Fig. 7. This data confirms a change in gene expression between the flat surface versus the fiber scaffold with a notable increase in expression of genes related to tenogenesis on the scaffold.

\section{Conclusions}

These results provide promising potential for the proposed scaffold design to be used as bioinstructive geometry. In consideration of the results, it is suggested that a 264-rpm $(0.087 \mathrm{~m} / \mathrm{s})$ scaffold could be advantageous for tendon

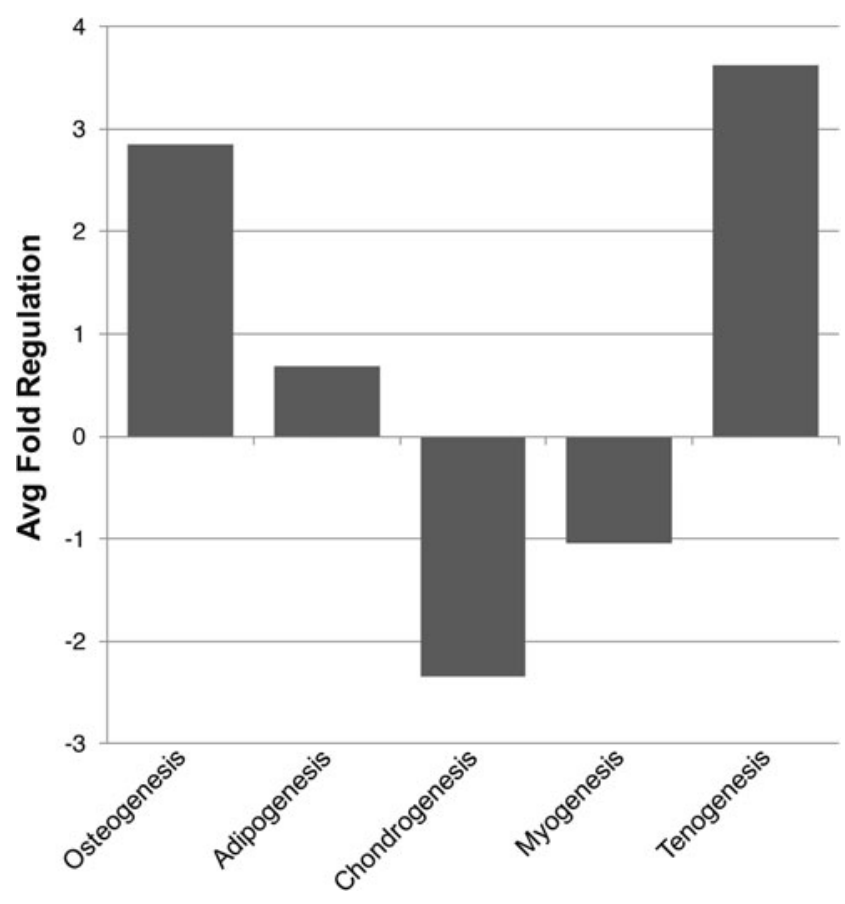

Fig. 7 Preliminary gene expression analysis: real-time PCR (RT-PCR) data presented as average fold regulation, cells seeded on glass coverslip served as the control surface, scaffold fiber diameter $=271 \mathrm{~nm}$

regenerative medicine applications based on the fiber architecture and corresponding toe region boundaries. Future directions include harnessing fiber architecture and diameter to align cells in an organized manner via the concept of bioinstructive geometry $[17,18]$ to replace or augment growth factors. The Chinese-fingertrap scaffold design provides compliance, an organized fiber matrix, and hollow tubular structure, suggesting the strategy as an encouraging approach for applications beyond tendon tissue engineering, including ligament engineering, vascular engineering [34], and nerve regeneration [35].

$P C L$ poly-(E-caprolactone), $h M S C s$ human mesenchymal stem cells, rpm rotations per minute, ECM extracellular matrix

Acknowledgments This material is based upon work supported by the National Institutes of Health grant R03AR065192 and the National Science Foundation under grant no. DGE1255832. Any opinions, findings, and conclusions or recommendations expressed in this material are those of the authors and do not necessarily reflect the views of the National Science Foundation. Special thanks to Gene Gerber and Andrew Higgins for assistance in the design and construction of the electrospinning mechanism as well as Dr. Jian Yang and Dr. Zhiwei Xie at the Pennsylvania State University for use of the tensile testing machine. Julie Anderson at the Materials Characterization Lab at the Pennsylvania State University is also recognized for her assistance in imaging the fiber samples.

\section{References}

1. Maffulli N, Renstrom P, Leadbetter WB, editors. Tendon injuries: basic science and clinical medicine. Springer; 2005. 
2. Chen J, Xu J, Wang A, Zheng M. Scaffolds for tendon and ligament repair: review of the efficacy of commercial products. Expert Rev Med Devices. 2009;6:61-73. doi:10.1586/174344440.6.1.61.

3. Martin B, Burr DB, Sharkey NA. Mechanical properties of ligament and tendon. In: Martin B, Burr DB, Sharkey NA, editors. Skelet. Tissue mech. New York: Springer; 1998. p. 309-47.

4. Lim JJ, Temenoff JS. Tendon and ligament tissue engineering: restoring tendon/ligament and its interfaces. In: Meyer U, Meyer T, Handschel J, Wiesmann HP, editors. Fundam. Tissue Eng. Regen. Med., Springer; 2009, p. 255-69. doi:10.1007/978-3-540-77755-7.

5. Galatz LM, Gerstenfeld L, Heber-Katz E, Rodeo SA. Tendon regeneration and scar formation: the concept of scarless healing. $\mathrm{J}$ Orthop Res. 2015;33:823-31. doi:10.1002/jor.22853.

6. Morais DS, Torres J, Guedes RM, Lopes MA. Current approaches and future trends to promote tendon repair. Ann Biomed Eng. 2015;43:2025-35. doi:10.1007/s10439-015-1369-5.

7. Kaeding CC, Aros B, Pedroza A, Pifel E, Amendola A, Andrish JT, et al. Allograft versus autograft anterior cruciate ligament reconstruction: predictors of failure from a MOON prospective longitudinal cohort. Sports Health. 2011;3:73-81. doi:10.1177/ 1941738110386185

8. Mason C, Dunnill P. A brief definition of regenerative medicine. Regen Med. 2008:3:1-5. doi:10.2217/17460751.3.1.1.

9. Laurencin CT, Khan Y. Regenerative engineering. Sci Transl Med. 2012;4:1-3. doi:10.1126/scitranslmed.3004467.

10. Laurencin CT, Daley GQ, James R. Regenerative engineering: materials, mimicry, and manipulations to promote cell and tissue growth. Bridg. 2013;43:34-41.

11. Zhang X, Bogdanowicz D, Erisken C, Lee NM, Lu HH. Biomimetic scaffold design for functional and integrative tendon repair. J Shoulder Elb Surg. 2012;21:266-77. doi:10.1016/j.jse. 2011.11.016.

12. Bosworth LA, Alam N, Wong JK, Downes S. Investigation of 2D and $3 \mathrm{D}$ electrospun scaffolds intended for tendon repair. J Mater Sci Mater Med. 2013;24:1605-14. doi:10.1007/s10856-013-4911-8.

13. James R, Kumbar SG, Laurencin CT, Balian G, Chhabra AB. Tendon tissue engineering: adipose-derived stem cell and GDF-5 mediated regeneration using electrospun matrix systems. Biomed Mater. 2011;6:1-13. doi:10.1088/1748-6041/6/2/025011.

14. Petrigliano FA, Arom GA, Nazemi AN, Yeranosian MG, Wu BM, McAllister DR. In vivo evaluation of electrospun polycaprolactone graft for anterior cruciate ligament engineering. Tissue Eng Part A. 2015;21:1228-36. doi:10.1089/ten.tea.2013.0482.

15. Xu Y, Wu J, Wang H, Li H, Di N, Song L, et al. Fabrication of electrospun poly(L-lactide-co-epsilon-caprolactone)/collagen nanoyarn network as a novel, three-dimensional, macroporous, aligned scaffold for tendon tissue engineering. Tissue Eng Part C. 2013;19:925-36. doi:10.1089/ten.TEC.2012.0328.

16. Jaiswal D, Brown JL. Nanofiber diameter-dependent MAPK activity in osteoblasts. J Biomed Mater Res Part A. 2012;100:2921-8. doi:10.1002/jbm.a.34234.

17. Ozdemir T, Xu L-C, Siedlecki C, Brown JL. Substrate curvature sensing through myosin IIa upregulates early osteogenesis. Integr Biol. 2013;5:1407-16. doi:10.1039/c3ib40068a.

18. Higgins AM, Banik BL, Brown JL. Geometry sensing through POR1 regulates Rac1 activity controlling early osteoblast differentiation in response to nanofiber diameter. Integr Biol. 2015;7:22936. doi:10.1039/C4IB00225C.
19. Maganaris CN, Paul JP. In vivo human tendon mechanical properties. J Physiol. 1999;521:307-13. doi:10.1111/j.1469-7793.1999. 00307.x.

20. Kuo CK, Marturano JE, Tuan RS. Novel strategies in tendon and ligament tissue engineering: advanced biomaterials and regeneration motifs. Sport Med Arthrosc Rehabil Ther Technol. 2010;2:114. doi:10.1186/1758-2555-2-20.

21. Hansen KA, Weiss JA, Barton JK. Recruitment of tendon crimp with applied tensile strain. J Biomech Eng. 2002;124:72-7. doi:10. 1115/1.1427698.

22. Donatelli R, Owens-Burkhart H. Effects of immobilization on the extensibility of periarticular connective tissue. J Orthop Sports Phys Ther. 1981;3:67-72. doi:10.2519/jospt.1981.3.2.67.

23. Eguchi A, Ochi M, Adachi N, Deie M, Nakamae A, Usman MA. Mechanical properties of suspensory fixation devices for anterior cruciate ligament reconstruction: comparison of the fixed-length loop device versus the adjustable-length loop device. Knee. 2014;21:743-8. doi:10.1016/j.knee.2014.02.009.

24. Su W-R, Chu C-H, Lin C-L, Lin C-J, Jou I-M, Chang C-W. The modified finger-trap suture technique: a biomechanical comparison of a novel suture technique for graft fixation. Arthrose J Arthrosc Relat Surg. 2012;28:702-10. doi:10.1016/j.arthro.2011.10.014.

25. Chung N-S, Cho J-H, Han K-J, Han S-H, Lee D-H. Tendon trap technique for rotator cuff repair. Orthopedics. 2012;35:1035-8. doi: 10.3928/01477447-20121120-04.

26. Ricker ZH, Rochat MC, Payton ME. Biomechanical evaluation of finger trap suture variants for securing catheters. J Am Vet Med Assoc. 2015;246:515-21. doi:10.2460/javma.246.5.515.

27. Yang D, Lu B, Zhao Y, Jiang X. Fabrication of aligned fibrous arrays by magnetic electrospinning. Adv Mater. 2007;19:3702-6. doi:10.1002/adma.200700171.

28. Liu Y, Zhang X, Xia Y, Yang H. Magnetic field-assisted electrospinning of aligned straight and wavy polymeric nanofibers. Adv Mater. 2011;22:2454-7. doi:10.1002/adma.200903870.

29. Maganaris CN, Narici M V. Mechanical properties of tendons. In: Maffulli N, Renström P, Leadbetter W, editors. Tendon Inj. Basic Sci. Clin. Med., Springer; 2005, p. 14-21.

30. Wang JH-C. Mechanobiology of tendon. J Biomech. 2006;39: 1563-82. doi:10.1016/j.jbiomech.2005.05.011.

31. Peniston SJ, Burg KJL, Shalaby SW. Design of abdominal wall hernioplasty meshes guided by mechanobiology and the wound healing response. In: Nagatomi J, editor. Mechanobiol. Handb., CRC Press; 2011, p. 499-514.

32. Domingos M, Dinucci D, Cometa S, Alderighi M, Bártolo PJ, Chiellini F. Polycaprolactone scaffolds fabricated via bioextrusion for tissue engineering applications. Int J Biomater. 2009;2009:1-9. doi: $10.1155 / 2009 / 239643$.

33. Lo H-Y, Kuo H-T, Huang Y-Y. Application of polycaprolactone as an anti-adhesion biomaterial film. Artif Organs. 2010;34:648-53. doi:10.1111/j.1525-1594.2009.00949.x.

34. Mitchell SL, Niklason LE. Requirements for growing tissueengineered vascular grafts. Cardiovasc Pathol. 2003;12:59-64. doi:10.1016/S1054-8807(02)00183-7.

35. Gu X, Ding F, Yang Y, Liu J. Construction of tissue engineered nerve grafts and their application in peripheral nerve regeneration. Prog Neurobiol. 2011;93:204-30. doi:10.1016/j.pneurobio.2010. 11.002. 